\title{
Towards accurate models for amorphous GeTe: Crucial effect of dispersive van der Waals corrections on the structural properties involved in the phase-change mechanism
}

\author{
M. Micoulaut, ${ }^{1}$ A. Piarristeguy, ${ }^{2}$ H. Flores-Ruiz, ${ }^{1}$ and A. Pradel ${ }^{2}$ \\ ${ }^{1}$ Paris Sorbonne Universités - UPMC, Laboratoire de Physique Théorique de la Matière Condensée, \\ Boite 121, 4 Place Jussieu, 75252 Paris Cedex 05, France \\ ${ }^{2}$ Institut Charles Gerhardt, UMR 5253-CNRS, Case 1503, Université de Montpellier, Place Eugène Bataillon, \\ 34095 Montpellier Cedex 5, France
}

(Received 21 May 2017; revised manuscript received 2 October 2017; published 17 November 2017)

\begin{abstract}
The effect of van der Waals dispersion correction in combination with density functional theory is investigated on a canonical amorphous phase-change material. Density functional theory (DFT), using the generalized gradient approximation, usually fails to reproduce the structure of amorphous tellurides, which manifests by an overestimation of the interatomic bond distances, and particularly the Ge-Te one involved in local geometries (tetrahedral or defect octahedral). Here, we take into account dispersion forces in a semiempirical way and apply such DFT simulations to amorphous GeTe. We obtain a substantial improvement of the simulated structure factor and pair-correlation function, which now reproduce the experimental counterparts with an unprecedented accuracy, including on a recent partial contribution from anomalous x-ray scattering and from x-ray absorption. A detailed analysis of the corresponding structures indicates that the dispersion correction reduces the $\mathrm{Ge}-\mathrm{Te}$ bond length, increases the fraction of tetrahedral germanium, and reduces the presence of heteropolar so-called fourfold $\mathrm{ABAB}$ rings. Given that these structural features have been stressed to be central for the understanding of the phase-change mechanism, the present results challenge our current understanding of the crystal to amorphous transformation at play.
\end{abstract}

DOI: 10.1103/PhysRevB.96.184204

\section{INTRODUCTION}

Rewritable optical devices have become the common media for data storage in modern information technologies, and the original idea traces back to the pioneering contributions of Ovshinsky in the late 1960s on phase-change (PC) phenomena in amorphous tellurides [1]. These materials not only display a certain number of remarkable properties regarding the switching between a crystalline and an amorphous phase, but are now also seen as promising candidates for other applications in optoelectronics such as digital versatile random access memory devices, blu-ray technology [2,3], or even fibers working at the long infrared wavelength for spatial interferometry [4], biosensing, or environmental metrology [5].

On PC properties and recording, a special emphasis is made on short crystallization times, on the optical or electrical contrast between the amorphous and the crystalline phase [2], on the reversibility between both phases upon a large number of phase-change cycles, and on the high thermal stability. Over the years, extensive investigations as a function of composition or alloying elements have led to the recognition that such important properties and performances are being optimized [6] along the $\mathrm{GeTe}-\mathrm{Sb}_{2} \mathrm{Te}_{3}$ in the ternary Ge-Sb-Te system (GST). In this search for optimal performances, the key material has turned out to be GeTe or $\mathrm{Ge}_{2} \mathrm{Sb}_{2} \mathrm{Te}_{5}$, one of the very first PC materials used for enhanced storage capacities [7] close to the $100 \mathrm{~GB} / \mathrm{cm}^{2}$ domain.

It is widely accepted that the enhanced optical and electrical contrast between the amorphous and crystalline phase of such materials results from an important difference in structure at the local scale, and particularly regarding the germanium atoms [6,8]. Early studies have suggested that this PC mechanism is, indeed, driven by a change in short-range order since $\mathrm{x}$-ray absorption fine structure (EXAFS) revealed that the Ge-Te bond length exhibits a significant reduction by about $0.22 \AA$ upon amorphization [9]. The reduction was explained on the basis of a simple umbrella flip model, suggesting that during the $\mathrm{PC}$ operation, the Ge atom switches from an octahedral site in the crystalline state to a fourfold tetrahedral site in the amorphous state. In fact, one of the basic features of tetrahedral-to-octahedral conversions driven by external conditions (light, current, pressure) is an increase of the atomic bond lengths $[10,11]$, which appears to be a natural mechanism in order to accommodate the electronic repulsion induced by a reduction of the bond angle from $109^{\circ}$ to about $90^{\circ}$. However, this simple and attractive model has been challenged by a series of experimental and theoretical studies. First, the umbrella flip model neglects the possibility to have homopolar Ge-Ge, and only Ge-Te bonds were considered. Such homopolar bonds were suggested to be present, as determined from another EXAFS study on another system $\left(\mathrm{Ge}_{2} \mathrm{Sb}_{2} \mathrm{Te}_{5}\right)$ [12]. Also, from a series of density functional theory (DFT)-based simulations [13-19], the defective octahedral geometry reminiscent of the crystalline rocksalt phase was found to be the dominant motif encountered in the amorphous phase. The determination of the population of tetrahedral Ge fraction has turned out to be a crucial quantity [20-25] in this respect, given that this structural motif involves a different chemical bonding reflected by different electronic orbitals. Indeed, $\mathrm{sp}^{3}$ tetrahedral geometries involve occupied but energetically unfavorable $\mathrm{sp}^{3}$ antibonding states and lead to the absence of resonance bonding, which is suggested [26] to control certain electronic properties for PC applications. This seems consistent with the fact that the ease of phase switching is directly linked to small ionicity and a limited degree of hybridization, enabling resonance p-electron bonding to prevail. However, recent materials exhibiting tetrahedral ordering have been found to exhibit PC properties as well [27]. 
The investigation [28,29] of the structure from x-ray (XRD) and neutron diffraction (ND) has also indicated that octahedral order dominates in the liquid phase. This has been also claimed [30] from the inspection of reciprocal space properties for a series of systems exhibiting changing tetrahedral to octahedral ordering. Specifically, an order parameter $S=S\left(k_{2}\right) / S\left(k_{1}\right)$ is defined from the amplitude $S\left(k_{2}\right)$ and $S\left(k_{1}\right)$ of the principal peaks of the structure factors. This permits distinguishing between an octahedral structure $(S<1)$, as in liquid GeTe, from a tetrahedral one $(S>1)$, as in germania [11] or $\mathrm{GeSe}_{2}$ [31]. In contrast with the latter, it is also important to emphasize that ND and XRD do not provide a full account of the structural correlations such as partial pair-correlation functions, essentially because of a weak contrast between the form factors and/or scattering lengths. Reverse Monte Carlo (RMC) models can fit the experimental functions [paircorrelation functions $g(r)$ and structure factor $S(k)$ ] quite accurately and are used in some cases as starting structural configurations for subsequent DFT investigations [22].

When such DFT modeling [13-19] of amorphous structures is obtained by performing a quench from the melt, an obvious lack of agreement with experiments is obtained. One of the major drawbacks is, indeed, an overestimation of the Ge-Te bond length (about 2.77-2.78 $\AA$ ) when the most usual Perdew-Burke-Ernzerhof (PBE) exchange-correlation functional is used, a feature which is known in the literature as the bond distance problem [22]. In fact, EXAFS measurements rather suggest an average Ge-Te bond length of $2.61 \pm 0.01 \AA$, such as in $\mathrm{Ge}_{2} \mathrm{Sb}_{2} \mathrm{Te}_{5}$ and $\mathrm{Ge}_{1} \mathrm{Sb}_{2} \mathrm{Te}_{4}$ [12,28], and a certain number of alternative artificial strategies [22,32,33] have been developed in order to increase the accuracy of the structural models. Also, the systematic overestimation of the Ge-Te bond lengths from DFT leads to a significantly higher fraction of octahedra, as quantitatively inferred from a study of amorphous Ge-Te alloys [34]. Given the proposed importance of these geometrical motifs and their underlying p-bond-related electronic properties for the crystal-amorphous transition, one thus arrives to the obvious conclusion that the bond distance problem is the major roadblock for a full understanding of the PC mechanism in tellurides.

At present, given these discrepancies, it is thus not surprising that there is still an active debate about the structure of such amorphous PC materials. From a theoretical viewpoint, possible improvements of structural models can emerge from the consideration of alternative DFT exchange-correlation functionals or even hybrid functionals. Recently, Caravati and Bernasconi [35] have used the Becke-Lee-Yang and Parr (BLYP) functional in order to investigate the structure of amorphous $\mathrm{Ge}_{2} \mathrm{Sb}_{2} \mathrm{Te}_{5}$. This functional is known to reduce the metallic character of the covalent bonding and provides an increased agreement with experiments in lighter chalcogenides [36-38]. The application has shown weak changes with respect to PBE, although a slight increase of the Ge tetrahedra fraction was acknowledged, also confirmed by an independent study from Kim et al. [39] which also led to a better agreement of the Ge-Te bond lengths with experimental results. A particular composition in the Ge-Te binary $\left(\mathrm{GeTe}_{4}\right)$ has been also investigated with different electronic schemes, and the main outcome was an increased accuracy once the BLYP functional was utilized [40].
In the present contribution we follow the same strategy, that is, a search for improved structural models for amorphous GeTe that can compare more favorably to experimental results from $\mathrm{x}$-ray diffraction, prior to an investigation of other properties. Rather than investigating the effect of an alternative functional, here we use the PBE one but by adding the semiempirical Grimme correction (DFT-D2) of van der Waals type [41]. Recent applications to the crystalline phase of $\mathrm{Ge}_{2} \mathrm{Sb}_{2} \mathrm{Te}_{5}$ has shown that the DFT-D2 method can lead to lattice parameters which are very close to the experimental ones [42], whereas the electronic properties and the band gap are also found [43] to be in excellent agreement with experiments. We, furthermore, build on a recent study of the liquid phase using such van der Waals schemes [44,45]. A direct comparison of simulated DFT-D2 Ge-Te [45] and Ge-Sb-Te liquids [46] with different compositions has shown, indeed, that the agreement with results from neutron diffraction was increased. Structural properties in real and reciprocal space were found to be reproduced with an excellent accuracy, and resulting coordination numbers and bond lengths were found to be reduced as compared to standard DFT, while also leading to an increased fraction of tetrahedra in the amorphous phase [34] for selected compositions in the Ge-Te binary. One may therefore wonder how such alternative schemes impact the structural properties of the amorphous phase of GeTe, which is one of the most promising PC materials, and a canonical system of interest for the basic understanding of PC properties. This is the purpose of the present paper that is in line with a previous application to the amorphous and liquid phase of $\mathrm{Ge}_{x} \mathrm{Te}_{100-x}$ with $x \leqslant 20 \%[34,45]$.

We find that DFT-D2 improves the structural properties of the PC materials GeTe by reproducing the total x-ray structure factor $S(k)$ at a level of unprecedented accuracy. The absorption spectra (EXAFS) is also substantially improved, and also the partial decomposition from anomalous X-ray scattering, this comparison between theory and experiment having not been realized before. In real space, corresponding paircorrelation functions $g(r)$ are found to be also dramatically improved when compared to a regular DFT calculation, while also being consistent with certain features of models obtained from RMC simulations. The full analysis reveals the tendency sketched above, that is, the reduction of Ge-Te distances leads, indeed, to a profound change in local geometries that is characterized quantitatively from topological constraints. The calculated coordination numbers are found to be consistent with experimental measurements from EXAFS. In addition, the decrease of the bond distances modifies the ring statistics and leads also to a reduction of heteropolar ring motifs (ABAB squares), which are thought to act as seeds during the recrystallization process. Taken together, these results reveal the importance of dispersive interactions for the accurate modeling of phase-change tellurides, consistent with previous findings for glasses or liquids, and also modify the current view of the central structural features involved in the phase-change phenomenon.

\section{SIMULATION METHODS}

Three amorphous models were obtained by quenching previously equilibrated liquids obtained at $820 \mathrm{~K}$ following a 
TABLE I. Elaboration and investigation characteristics of GeTe amorphous films studied by different research groups [29,48-50]. The information marked with an asterisk $(*)$ corresponds to deposition of Ge-Sb-Te amorphous films performed by the group of Yamada and Matsunaga [51].

\begin{tabular}{|c|c|c|c|}
\hline Film Elaboration Technique & Thickness & Composition & XRD experiment \\
\hline $\begin{array}{l}\text { Sputtering [29] from a stoichiometric GeTe } \\
\text { target on a glass disk substrate.* }\end{array}$ & $\simeq 1 \mu \mathrm{m}^{*}$ & $\begin{array}{l}\text { Checked by inductively coupled } \\
\text { plasma spectrometry.* }\end{array}$ & $\begin{array}{l}\text { High-energy XRD on beamline BL02B2 } \\
\text { and BL04B2 at the SPring- } 8 \text { (Hyogo, } \\
\text { Japan). }\end{array}$ \\
\hline $\begin{array}{l}\text { Plasma-assisted sputtering [48] from a } \\
\text { stoichiometric GeTe target. }\end{array}$ & $\simeq 200 \mathrm{~nm}$ & - & $\begin{array}{l}\text { High-energy XRD on beamline CRISTAL } \\
\text { at the SOLEIL Synchrotron (Saclay, } \\
\text { France) using a 2D image plate detector. }\end{array}$ \\
\hline $\begin{array}{l}\text { dc magnetron sputtering [49] from a } \\
\text { stoichiometric GeTe target on Si wafers } \\
\text { coated with PMMA. }\end{array}$ & $\simeq 1-3 \mu \mathrm{m}$ & $\begin{array}{l}\text { Checked by energy-dispersive } \\
\text { x-ray analysis. }\end{array}$ & $\begin{array}{l}\text { High-energy XRD at the BW5 Wiggler } \\
\text { beamline of the DORISIII positron } \\
\text { storage ring operated at the } \\
\text { HASYLAB/DESY (Hamburg, } \\
\text { Germany). }\end{array}$ \\
\hline $\begin{array}{l}\text { Radio-frequency sputtering [50] from a } \\
\text { stoichiometric GeTe target on silica } \\
\text { substrate. }\end{array}$ & $\simeq 1 \mu \mathrm{m}$ & $\begin{array}{l}\text { Checked by energy dispersive } \\
\text { X-ray analysis. }\end{array}$ & $\begin{array}{l}\text { Standard } \omega-2 \theta \text { diffractometer installed at } \\
\text { the beamline BM02 at the ESRF } \\
\text { (Grenoble, France) or/and beamline } \\
\text { BL13XUof at the SPring- } 8 \text { (Hyogo, } \\
\text { Japan) using two incident X-ray energies } \\
\text { ( }-20 \text { and }-200 \mathrm{eV} \text { for } \mathrm{Ge},-30 \mathrm{eV} \text { and } \\
-300 \mathrm{eV} \text { for Te, respectively) below the } \\
K \text { absorption edges of each constituent } \\
\text { element. }\end{array}$ \\
\hline
\end{tabular}

quenching procedure developed in Ref. [34]. These were found to reproduce very accurately the measured pair-correlation functions and structure factors for somewhat different compositions [29,48-50]. Molecular dynamics (MD) simulations of Car-Parrinello type (CPMD code) were performed on a 200atom system in a cubic box with periodic boundary conditions and a density equal to experimental ones $\left(0.0337 \AA^{-3}\right)$ [29]. The electronic structure has been described within DFT, and evolved self-consistently during the motion. A generalized gradient approximation (GGA) has been used, and valence electrons were treated explicitly, in conjunction with TroullierMartins norm-conserving pseudopotentials. The wave functions were expanded at the $\Gamma$ point of the supercell on a plane-wave basis set with an energy cutoff of 20 Ry. During the CPMD simulation, a fictitious electron mass of 2000 a.u. and a time step of $0.12-0.36$ fs was used to integrate the equations of motion. Three models of amorphous GeTe were considered, a first using the standard PBE functional as in Refs. [13-19] (termed PBE hereafter), a second one based on an improved scheme for the exchange-correlation energy obtained by Perdew, Burke, and Ernzerhof (termed PBEsol hereafter) [47], and a third one coupling the latter with the inclusion of dispersion forces (termed vdW hereafter). The PBEsol functional has been used previously for the investigation of a GST model [52], and it was stated that PBEsol improves the bond length with respect to PBE. However, in this particular contribution the PBEsol model was not obtained from the melt but from a $300 \mathrm{~K}$ equilibration of a RMC model. It is, therefore, tempting to probe the effect of three different simulation schemes on a material having undergone the same thermal history. We stress at this point that the pseudopotentials used (PBE, PBEsol) belong to the same pseudopotential library as the one used in Ref. [20]. As discussed below, results slightly differ, however, and might be related to the way we obtained the amorphous systems. For each model, three independent sets of atomic positions were, indeed, chosen from the equilibrated $820 \mathrm{~K}$ trajectory and used as starting configurations for the quenching procedure after an additional run of $25 \mathrm{ps}$ at $820 \mathrm{~K}$. The systems were first quenched to $600 \mathrm{~K}$ for $25 \mathrm{ps}$, prior to a second quench to $300 \mathrm{~K}$, that was performed over 3 ps. The first picoseconds were discarded, and the whole trajectory of 40 ps was used for statistical analysis. Temperature control was implemented for both the ionic and electronic degrees of freedom by using Nosé-Hoover thermostats with a frequency of $800 \mathrm{~cm}^{-1}$. During the whole simulation, the attractive dispersion (Grimme) correction [41] was taken into account, and corresponding parameters can be found in Ref. [44].

\section{RESULTS}

\section{A. A review of select experimental measurements}

Before comparing the obtained simulated structure functions in real and reciprocal space, it is important to contrast and compare the experimental findings because a certain number of differences appear, and these also reflect the preparation methods.

Several research groups [29,48-50] have been studying the structure of GeTe amorphous films in the last ten years. The films were elaborated by either radio frequency or $\mathrm{dc}$ magnetron sputtering from a stoichiometric GeTe target. The thickness of the deposited films ranged between $200 \mathrm{~nm}$ and $3 \mu \mathrm{m}$ approximately (Table I). The composition of the 


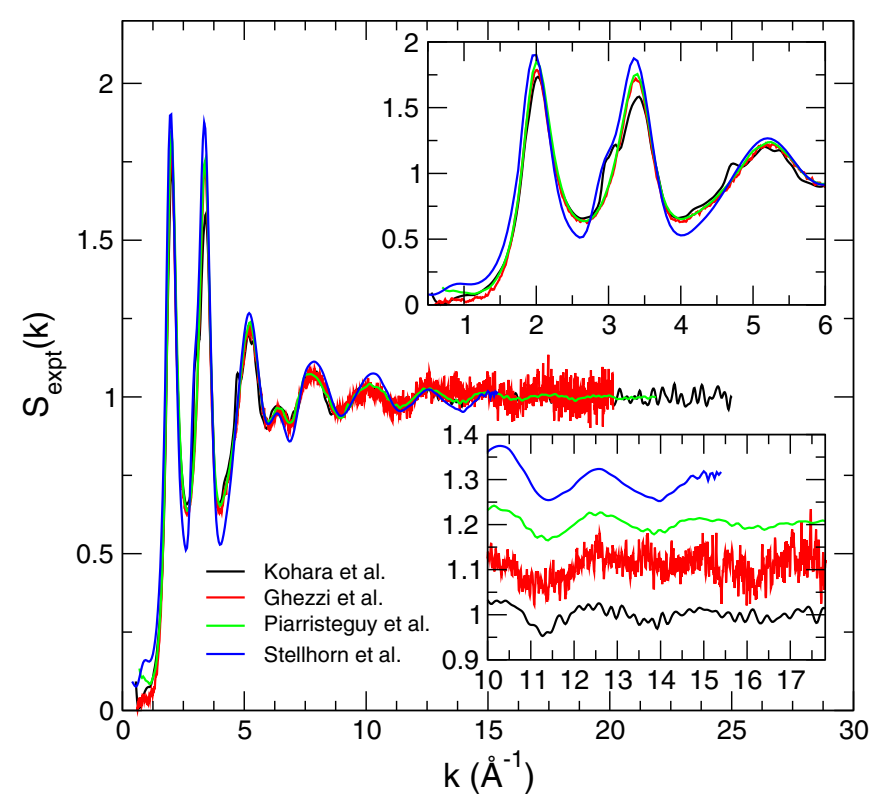

FIG. 1. A comparison of the total structure factor $S_{\text {expt }}(k)$ measured by Kohara et al. [29], Ghezzi et al. [48], Piarristeguy et al. [49], and Stellhorn et al. [50]. The top inset shows the low-wave-number region and highlights the three principal peaks, whereas the bottom inset represents the long-wave-number domain (structure functions have been shifted).

films (except in one case [48]) was checked by inductively coupled plasma (ICP) spectrometry or by energy-dispersive $\mathrm{X}$-ray (EDX) analysis. X-ray diffraction experiments were performed on different large-scale facilities [SPring-8 (Hyogo, Japan), SOLEIL (Saclay, France), HASYLAB/DESY (Hamburg, Germany), ESRF (Grenoble, France)]. A careful look at the experimental structure factors of the GeTe amorphous films shows some differences which cannot be overlooked, especially when the data are used as the data to be fitted for RMC modeling $[29,49,50]$.

The different measured total structure factors $S_{\text {expt }}(k)$ are shown in Fig. 1. In all cases, five main oscillations can be identified in the low-wave-vector range $\left(k<9 \AA^{-1}\right)$. The $S_{\text {expt }}(k)$ reported in Refs. [48] and [49] are rather similar, while the data from Refs. [29] and [50] differ in a significant way. In particular, a shoulder at around $3 \AA^{-1}$, much more pronounced in the case of data from Ref. [29], is observed. The positions of the two principal peaks (PPs) at $k_{1}$ and $k_{2}$ change slightly depending on the experiments. The first peak position $k_{1}$ shifts from 1.97 to $2.02 \AA^{-1}$, and the position of the second PP $k_{2}$ ranges between 3.36 and $3.42 \AA^{-1}$ (see inset Fig. 1). In fact, the main difference between the structural factors $S_{\text {expt }}(k)$ arises from the difference in amplitudes between the two peaks, $S\left(k_{2}\right)$ and $S\left(k_{1}\right)$, which can be analyzed on the basis of parameter $S=$ $S\left(k_{2}\right) / S\left(k_{1}\right)$. The calculated values of parameter $S$ are reported in Table II. While $S$ is lower than 1 for all the experiments, its values change significantly between 0.79 and 0.97 , depending upon the experiments. Finally, both the main Fig. 1 and the inset at the bottom-right-end side of the figure clearly show a better resolution for data from Ref. [50], which could indicate
TABLE II. Measured positions, $k_{1}$ and $k_{2}$, of the first two principal peaks of the total structure factors $S_{\text {expt }}(k)$ and order parameter $S=$ $S\left(k_{2}\right) / S\left(k_{1}\right)$ of GeTe amorphous films studied by different research groups [29,48-50].

\begin{tabular}{lccc}
\hline \hline Reference & $k_{1}\left(\AA^{-1}\right)$ & $k_{2}\left(\AA^{-1}\right)$ & $S=S\left(k_{2}\right) / S\left(k_{1}\right)$ \\
\hline$[29]$ & 2.02 & 3.42 & 0.79 \\
{$[48]$} & 2.01 & 3.38 & 0.92 \\
{$[49]$} & 2.00 & 3.39 & 0.88 \\
{$[50]$} & 1.97 & 3.36 & 0.97 \\
\hline \hline
\end{tabular}

a much better ordered structure for the films obtained in this case.

The present compilation exemplifies how different preparation conditions (Table I) influence atomic correlations at longrange order, as highlighted by the distribution of principal peak positions and amplitudes. In addition, the effect of thickness is known to affect substantially the local structural order of amorphous tellurides. Numerical simulations have shown, indeed, that for $\mathrm{Ge}_{2} \mathrm{Sb}_{2} \mathrm{Te}_{5}$ as-deposited materials show a significantly larger fraction of homopolar bonds $(\mathrm{Ge}-\mathrm{Ge})$ and consequently, a larger fraction of tetrahedra [53] when compared to melt-quenched models (27\%) [52]. One obtains a fraction of Ge atoms in a tetrahedral geometry, about $54 \%$.

\section{B. Simulated total structure factor}

Having such experimental data in hand, we first concentrate on the simulated reciprocal space properties. Figure 2 exhibits the calculated function $S_{X}(k)$ corresponding to the x-ray weighted total structure factor, defined from the partials $S_{i j}(k)$, via

$$
S_{X}(k)=\frac{\sum_{i, j} x_{i} x_{j} f_{i} f_{j}\left(1+\delta_{i j}\right) S_{i j}(k)}{\sum_{i, j} x_{i} x_{j} f_{i} f_{j}},
$$

where $f_{i}$ and $f_{j}$ represent atomic form factors of atom $i$ and $j$, respectively. It is generally assumed that these do not depend on spatial frequency so that the high-energy limit is usually assumed, i.e., $f_{i}$ is replaced by atomic numbers (Ge:32, Te:52). The partials have been calculated from the trajectories using

$$
S_{i j}(k)=\frac{1}{N}\left\langle\sum_{k=1}^{N_{i}} \sum_{l \neq k}^{N_{j}} \exp \left[-k\left(\mathbf{r}_{k}-\mathbf{r}_{l}\right]\right\rangle .\right.
$$

An inspection of Fig. 2 shows that the agreement with experimental results from XRD is excellent once the dispersion forces are taken into account. Here we have compared our calculated $S_{X}(k)$ with those measured by Piarristeguy et al. [49]. For clarity, the other data have not been represented. Readers can refer to Fig. 1 for an additional comparison.

Results indicate that the absence of dispersion corrections (e.g., PBEsol) leads to a reduced amplitude for both principal peaks obtained at $k_{1}=2.0$ and $k_{2}=3.45 \AA^{-1}$ (green curve in Fig. 1), the third peak at $k_{3}=5.2 \AA^{-1}$ being nearly unaffected by the two numerical schemes. In addition, a typical peak at $\simeq 6.2 \AA^{-1}$ is also found to be much better reproduced (Fig. 3, virtually absent without the DFT-D2 scheme), although we also note that both simulation schemes lead to a correct 


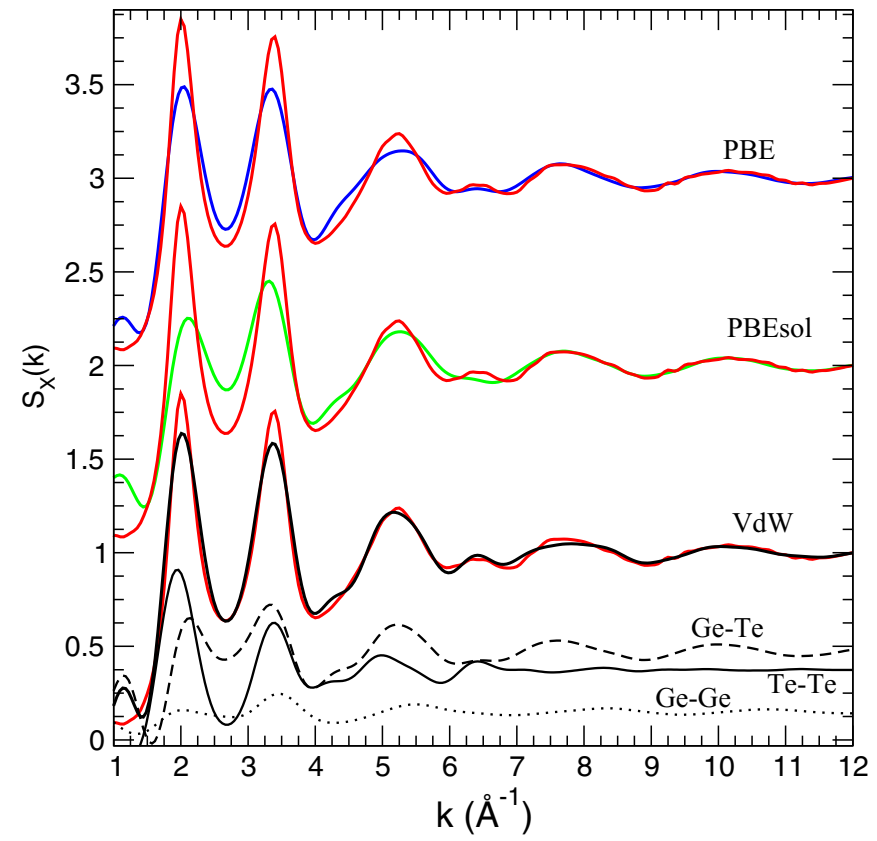

FIG. 2. Calculated (vdW, black) XRD structure factor $S_{X}(k)$ of amorphous GeTe compared to experimental results from Piarristeguy et al. [49] (red curves, duplicated). The green and blue curves represent results from a simulation without dispersion correction and correspond to PBEsol and PBE schemes, respectively. The black lines correspond to the decomposition into partials $x_{i} x_{j} f_{i} f_{j} S_{i j} /\langle f\rangle^{2}$ for the vdW case.

reproduction of large- $k$ oscillations that are visible once the interference $I(k)=k\left[S_{X}(k)-1\right]$ is represented (Fig. 3). Both PBE and PBEsol simulations, furthermore, lead to a tail at $4.5 \AA$ that is not observed experimentally. A decomposition

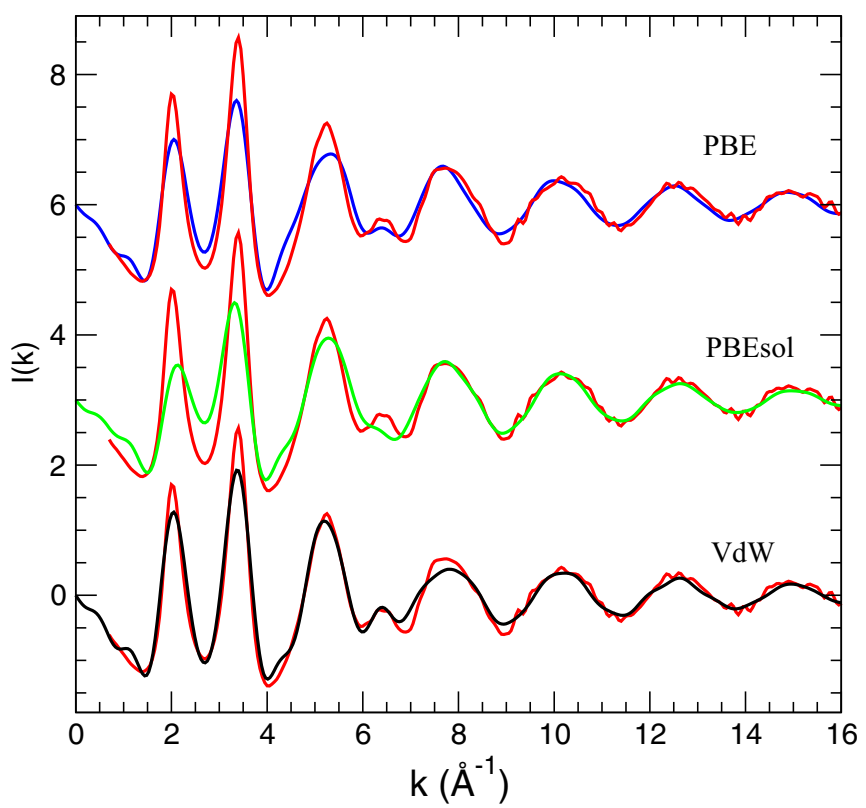

FIG. 3. Calculated XRD interference function for the vdW (black), PBEsol (green), and PBE (blue) simulations, compared to experimental data (red, duplicated) from Ref. [49].

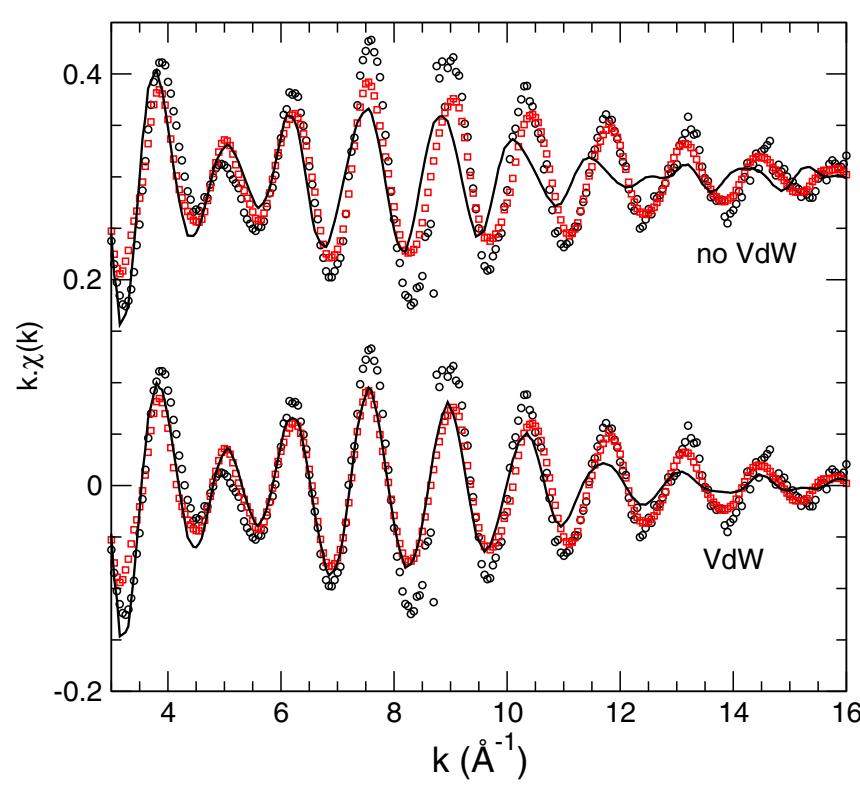

FIG. 4. Computed EXAFS spectra of amorphous GeTe (vdW and PBEsol without vdW correction), compared to absorption results from Noé et al. (black circles [57]) and Van Eijk (red squares [58]).

into partial structure factors (Fig. 2) indicates that all improved features [increased contrast of between $S\left(k_{1}\right)$ and $S\left(k_{2}\right)$, small peak at $6.2 \AA^{-1}$ ] are due to the Te-Te partials which contain such features, while being also dominated by Ge-Te correlations at $k_{1}, k_{2}$, and $k_{3}$.

\section{X-ray absorption}

This improved structural description in reciprocal space is also visible from the EXAFS quantity $k \cdot \chi(k)$ that has been calculated using the multiple-scattering theoretical approach [54], which has been shown to reproduce accurately the experimental spectra of many crystals and appropriately extended to the study of liquid and amorphous systems [55], while also recently used for PC materials [56] including for the near-edge part. When this framework is applied to the simulated GeTe compounds, results can be directly compared to experimental data from x-ray absorption spectroscopy (XAS) experiments at the Ge $K$-edge $(11103 \mathrm{eV})$. Figure 4 shows the calculated spectrum of amorphous GeTe for both simulation schemes, which is compared to experimental data from Noé et al. (black circles [57]) and Van Eijk [58] (red squares). The EXAFS spectrum is found to be substantially improved here using the Grimme corrections (vdW) given that the typical oscillations $k \cdot \chi(k)$ can be reproduced over a broad range of considered wave vectors $\left(3-16 \AA^{-1}\right)$, whereas the absence of such corrections obviously leads to a phase lag for wave vectors larger than $5 \AA^{-1}$.

\section{Comparison with a partial AXS analysis}

It is interesting to compare the present simulated data with very recent results [50] from anomalous X-ray scattering (AXS), which permits one to distinguish the partial contributions of both $\mathrm{Ge}$ and $\mathrm{Te}$ by focusing on scattering close 


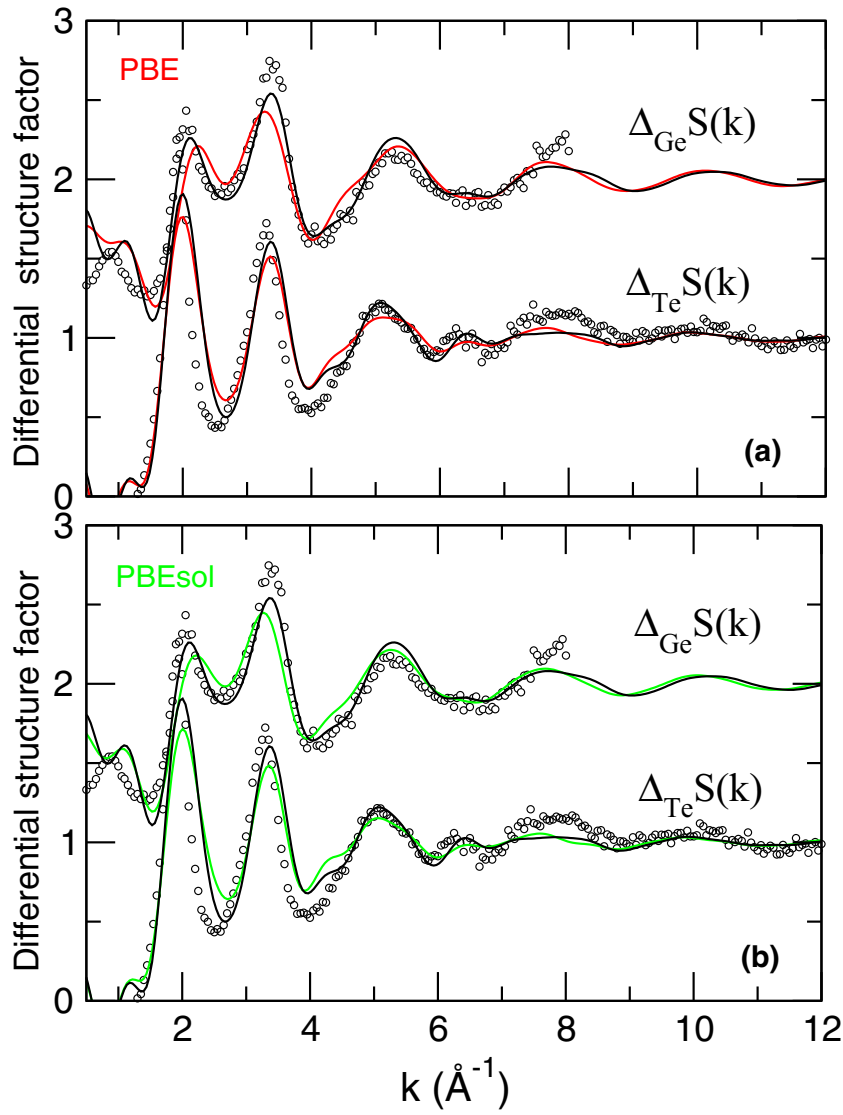

FIG. 5. (a) Calculated difference functions $\Delta_{\mathrm{Ge}} S(k)$ and $\Delta_{\mathrm{Te}} S(k)$ for amorphous GeTe for the vdW (black curves) and the PBE (red curves) simulations, compared to experimental data from AXS [50]. (b) Same quantities but for a comparison between vdW (black) and PBEsol (green).

to the $K$ edge. For DFT-based simulations of amorphous tellurides, it should be stressed that this kind of comparison has not been performed before, although it is well known that for network-forming species such as $\mathrm{As}_{2} \mathrm{Se}_{3}$ [59] or $\mathrm{GeSe}_{2}$ [60], a direct comparison provides an additional constraint for the validity of the obtained structural models. Once the anomalous variation of the atomic form factor is taken into account near an absorption edge, one can utilize the difference between two scattering spectra close to the absorption edge of a relevant species in order to obtain differential structure factors $[61,62]$,

$$
\Delta_{i} S(k)=\sum_{i} \sum_{j} W_{i j} S_{i j}(k),
$$

where the weighting factors $W_{i j}$ depend on the real and imaginary part of the anomalous form factor, and corresponding values can be found in Ref. [50].

Figure 5 represents the experimental measurements of $\Delta_{\mathrm{Ge}} S(k)$ and $\Delta_{\mathrm{Te}} S(k)$ (circles) together with those calculated from DFT and DFT-D2 using Eqs. (2) and (3). It is seen that such partial contributions are only fairly reproduced from the DFT model (PBE and PBEsol) and, for instance, the position of the secondary peak for both $\Delta_{\mathrm{Te}} S(k)$ and $\Delta_{\mathrm{Ge}} S(k)$ at $3.6 \AA^{-1}$ is shifted in wave number. Using DFT-D2, one can also notice

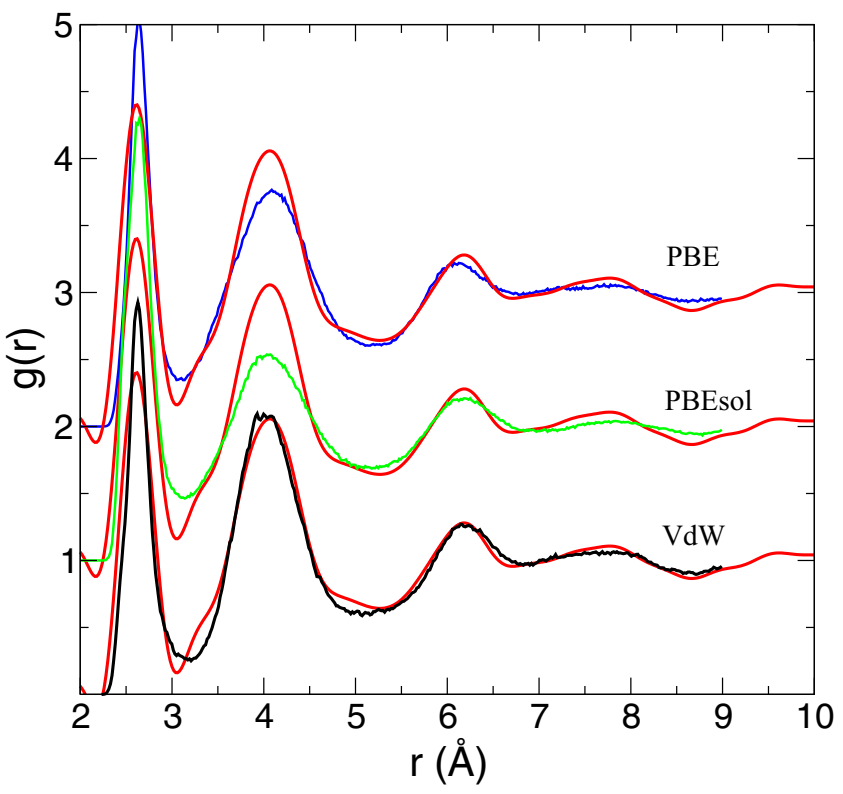

FIG. 6. Calculated total pair-correlation function $g(r)$ of amorphous GeTe (black) compared to results from XRD (red [48], duplicated) and a simulation without the dispersion correction: PBEsol (green, shifted) and PBE (blue, shifted).

that both $\Delta_{\mathrm{Ge}} S(k)$ and $\Delta_{\mathrm{Te}} S(k)$ are rather well reproduced in peak positions, widths, and amplitudes. The decomposition into partials permits one to infer an origin for certain features of the total structure factor $S(k)$. Indeed, the small first sharp diffraction peak (FSDP) observed at around $1 \AA^{-1}$ (both experimentally and theoretically, Fig. 1) is due to $\Delta_{\mathrm{Ge}} S(k)$, and this indicates an increased intermediate range ordering for the Ge atoms only. $\Delta_{\mathrm{Ge}} S(k)$ furthermore provides only a small contribution to the principal peak found at about $2.0 \AA^{-1}$, revealing that $\mathrm{Te}-\mathrm{Te}$ and $\mathrm{Ge}-\mathrm{Te}$ correlations are the dominant contributions to the principal peak, as already revealed from the full partial decomposition (Fig. 2). Similarly, the typical small peak at $\simeq 6.2 \AA^{-1}$ that is not reproduced with standard DFT (Fig. 3) arises from $\Delta_{\mathrm{Te}} S(k)$, as shown both from AXS [50] and DFT-D2 results.

\section{E. Properties of the real space}

We now represent in Fig. 6 the total pair-correlation function $g(r)$ weighted by using the XRD form factors $f_{i}$ given above. Once again, the effect of the dispersion correction induces an overall improvement of the structure because the experimental secondary peak at $4.1 \AA$ is now perfectly reproduced. It is also important to mention that the absence of such dispersive forces leads to a secondary peak with a smaller amplitude and to a first minimum (at $r_{m}=3.1 \AA$ ) that is less well defined. This indicates a reduced structuration of the corresponding amorphous phase, i.e., first and second shell neighbors are not well separated, in contrast with what is usually encountered in lighter chalcogenides where $g\left(r_{m}\right)=0$ (e.g., $\mathrm{GeSe}_{2}$ [63]). The partial analysis (Fig. 7) shows that the vdW simulations lead to minor changes in the global shape of the partial pair-correlation functions $g_{i j}(r)$ with respect 

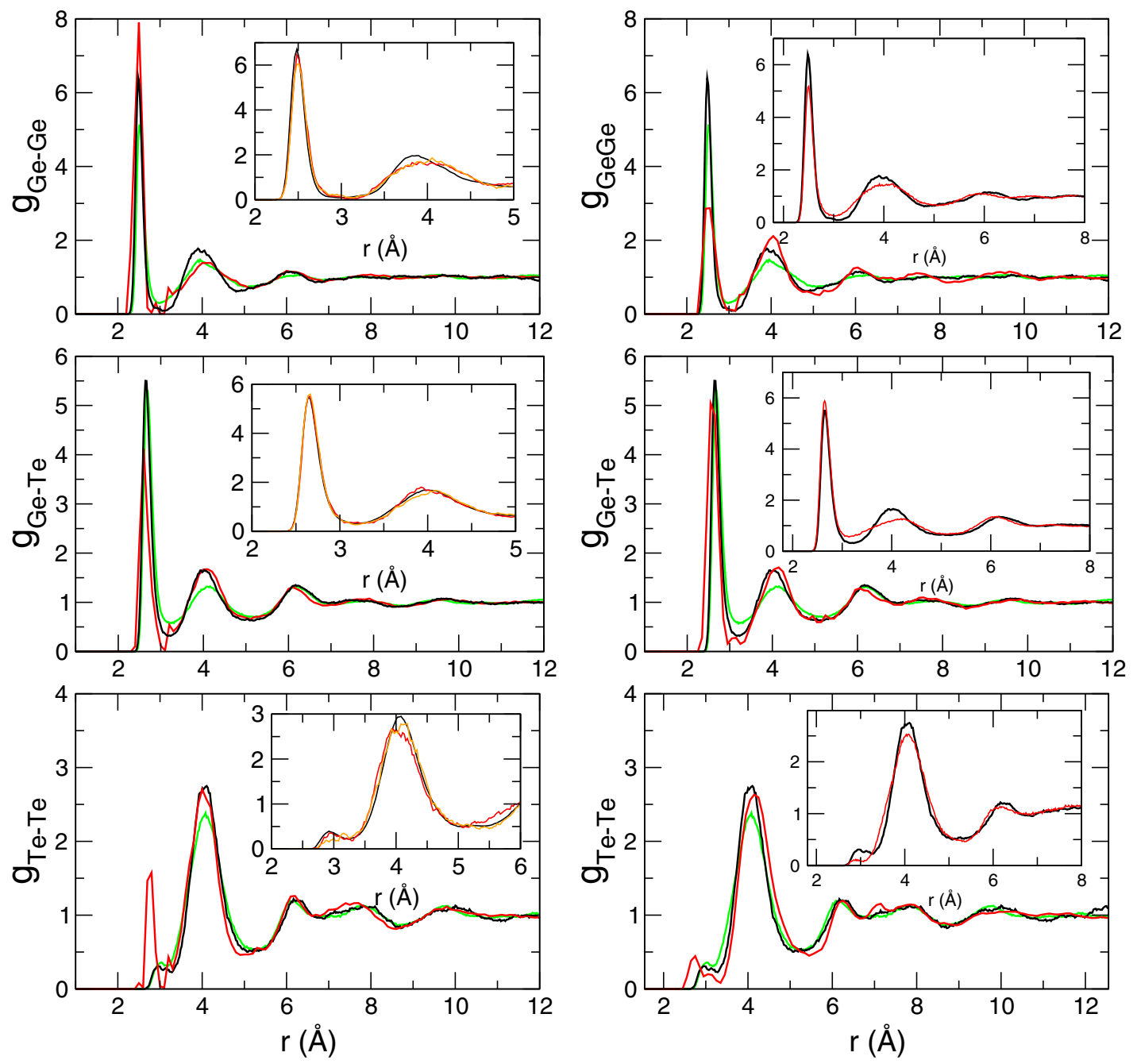

FIG. 7. Computed partial pair-correlation functions $g_{i j}(r)(i, j=\mathrm{Ge}, \mathrm{Te})$ in amorphous GeTe (vdW, black curves; PBEsol, green curves), compared to RMC results (red curves) from Piarristeguy et al. (left [49]) and Stellhorn et al. (right [50]). The insets on the left show the corresponding quantities for the three independent quenches for the vdW simulation. The insets on the right show a comparison between the vdW (black) and the PBE (red) simulations.

to PBE or PBEsol, particularly for the Ge-Te correlations, which turn out to be crucial for the structural accuracy of the model. Small but significant differences appear, however, and these (peak positions and amplitudes) turn out to be crucial for determining the accuracy of the corresponding models. It is also important to emphasize that these partial correlation functions $g_{i j}(r)$ display moderate fluctuations with respect to thermal history (Fig. 7 insets), some small differences being observable in the Te-Te correlations. As a result, the obtained numbers and bond distances only weakly depend on the local potential energy minimum in which the liquid has been trapped by the rapid quench of the simulation.

When compared to a previous study of amorphous GeTe using RMC models [49,50], Fig. 7 now also reveals that the DFTD2 simulation is globally consistent with the pair-correlation analysis obtained by different groups. Nevertheless, a notable difference is found in the Te-Te prepeak corresponding to residual homopolar defects. In the RMC simulation, the Te-Te homopolar correlations are still relatively abundant, whereas they are barely visible in DFT simulations and also from a visual inspection of the network structure. One should also note that the RMC fits by Stellhorn et al. [50] lead to an amplitude for the homopolar Ge-Ge peak at $\simeq 2.4 \AA$ that is significantly reduced.

\section{PROPERTIES OF THE DISPERSIVE GeTe MODELS}

So far, we have checked the overall quality of an improved structural model of amorphous GeTe using the van der Waalslike dispersive correction. Results for both the total structural factor $S_{X}(k)$ and the pair-correlation function $g(r)$ have been found to be of unprecedented accuracy when compared to corresponding experimental results. An additional comparison with the EXAFS spectrum and the partial structure factors from AXS has confirmed this overall conclusion. We now focus on a certain number of structural features that can be determined from the full analysis of the atomic trajectories obtained from the vdW simulations. 
TABLE III. Calculated bond distances $d_{i j}$ in amorphous GeTe from different modeling schemes. Note that the present $d_{\mathrm{TeTe}}$ have been determined from an inspection of the atomic structure, given the weak contribution (shoulder peak) to $g_{\mathrm{TeTe}}(r)$ (see Fig. 7).

\begin{tabular}{lccc}
\hline \hline & $d_{\mathrm{GeGe}}(\AA)$ & $d_{\mathrm{GeTe}}(\AA)$ & $d_{\mathrm{TeTe}}(\AA)$ \\
\hline DFT-D2 (vdW) & 2.48 & 2.66 & 2.89 \\
PBEsol & 2.51 & 2.69 & 2.89 \\
PBE & 2.50 & 2.70 & 2.89 \\
EXAFS [9] & & 2.61 & \\
EXAFS [57] & 2.47 & 2.62 & \\
EXAFS [64] & 2.48 & 2.65 & \\
EXAFS [58] & 2.47 & 2.61 & 3.00 \\
EXAFS [65] & & 2.59 & \\
RMC [50] & 2.53 & 2.60 & 2.73 \\
RMC [58] & 2.48 & 2.61 & \\
RMC [49] & 2.50 & 2.60 & 2.70 \\
DFT [20] & 2.54 & 2.78 & 2.85 \\
\hline \hline
\end{tabular}

\section{A. Reduced bond lengths}

An inspection of Table III shows that a global improvement of the bond distances is obtained because we find a Ge-Te bond length of $2.66 \AA$, i.e., slightly shorter than that obtained from the PBEsol simulation $(2.69 \AA)$, the latter value being close to the results obtained from PBE modeling schemes (2.70, present, or $2.78 \AA$ [20]). Except for the global agreement found for the total pair-correlation function and structure factor, the effect of the dispersive forces on local correlating distances (bond lengths) is small, and their inclusion seems to impact mostly second-neighbor correlating distances, as acknowledged by Fig. 6. The obtained value for $d_{\mathrm{GeTe}}$ is, however, more in line with diffraction data. Experimentally, it has been essentially measured $d_{\mathrm{GeTe}}=2.60-2.61 \AA[9,57,58,64,65]$, as also determined from RMC modeling [49,50,57,58]. In addition, the obtained $\mathrm{Ge}-\mathrm{Ge}$ correlating distance is found to be rather close to the one determined from EXAFS [12,58,64], which found $d_{\mathrm{Ge}-\mathrm{Ge}}=2.47-2.48 \AA$, and to $\mathrm{RMC}$ values $[50,58,66]$ obtained from a fit of both AXS and XRD results (2.48 $\AA-2.53 \AA)$. The distance of this bond type is found, indeed, to vary between $2.53 \AA$ [50] and $2.50 \AA$, the latter being fitted from both XRD and EXAFS [49]. On the Ge-Ge correlations, the effect of the van der Waals correction is seen to also cause a slight reduction of the homopolar $\mathrm{Ge}-\mathrm{Ge}$ bond distance from $2.51 \AA$ to $2.48 \AA$. The reduction of the bond distance usually has important consequences regarding the $\mathrm{Ge}$ geometry, as already stressed for other compositions in the GeTe binary and discussed below. Concerning the Te-Te distance, we find $d_{\mathrm{Te}-\mathrm{Te}} \simeq 2.89 \AA$, somewhat larger than the distance found from RMC models [49,50,58] (see prepeak in Fig. 7), in qualitative agreement with previous DFT calculations [20], and closer to the measured [58] $d_{\mathrm{Te}-\mathrm{Te}}=3.00 \AA$.

It should also be stressed that even the EXAFS data exhibit some differences, and this might be due to differences in measurement and sample conditions (temperature, $\mathrm{Ge}$ or $\mathrm{Te}$ edge, thickness, deposition technique). When the absorption experiment is performed at room temperature, only the largest peak is visible in the Fourier-transformed spectra [58] so
TABLE IV. Calculated coordination numbers $n_{i j}=n_{i j}\left(r_{m}\right)$ with $i, j=(\mathrm{Ge}, \mathrm{Te})$, total coordination number $n_{i}(i=\mathrm{Ge}, \mathrm{Te})$, and mean coordination number $\bar{r}$ in amorphous GeTe compared to experimental results, and to previous simulations using RMC and DFT. The number indicated in brackets corresponds to the minimum (cut-off distances) $r_{m}$ of the corresponding function $g_{i j}(r)$ and have been used to evaluate $n_{i j}$.

\begin{tabular}{lcccccc}
\hline \hline & $n_{\mathrm{GeGe}}$ & $n_{\mathrm{GeTe}}$ & $n_{\mathrm{TeTe}}$ & $n_{\mathrm{Ge}}$ & $n_{\mathrm{Te}}$ & $\bar{r}$ \\
\hline DFT-D2 (vdW) & 1.79 & 2.20 & 0.28 & 3.99 & 2.48 & 3.24 \\
& $(2.97)$ & $(3.20)$ & $(3.23)$ & & & \\
PBEsol & 1.71 & 2.45 & 0.23 & 4.16 & 2.68 & 3.42 \\
& $(2.97)$ & $(3.20)$ & $(3.18)$ & & & \\
PBE & 1.66 & 2.52 & 0.06 & 4.18 & 2.58 & 3.38 \\
& $(3.10)$ & $(3.18)$ & $(3.08)$ & & & \\
EXAFS [57] & $1.65-1.89$ & $1.38-1.47$ & & & & \\
EXAFS [64] & 1.2 & 2.5 & & & & \\
EXAFS [58] & 1.70 & 1.40 & 0.30 & & & \\
EXAFS [65] & & 1.5 & & & & \\
DFT [20] & 1.10 & 3.20 & 0.10 & 4.20 & 3.30 & 3.75 \\
RMC [49] & 2.45 & 1.50 & 0.60 & 3.95 & 2.10 & 3.03 \\
RMC [50] & 1.44 & 2.29 & 0.23 & 3.73 & 2.52 & 3.12 \\
\hline \hline
\end{tabular}

that only the two Ge-Ge and Ge-Te bond lengths could be measured.

\section{B. Coordination numbers compatible with experiments}

From the obtained simulated pair-correlation functions $g_{i j}(r)$ (Fig. 7), we obtain by integrating up to corresponding first minima $r_{m}$ (see Table IV) the partial coordination numbers $n_{i j}$ for the different models of amorphous GeTe using

$$
n_{i j}=4 \pi \rho \int_{0}^{r_{m}} r^{2} g_{i j}(r) d r .
$$

From such calculated coordination numbers $n_{i j}$, we then determine the coordination numbers of the species, which are given by $n_{i}=n_{i i}+n_{i j}$. One should first keep in mind that a discussion of such numbers is rather overrated given that the experimental determination of $n_{i j}$ depends substantially on the preparation conditions and aging behavior of the system [57], which leads, e.g., to a rather large domain of possible coordination numbers, e.g., for the Ge-Te pair (1.38-2.50).

From the calculation, it is first seen that the dominant contribution to Ge and Te coordination number arises from the connections with $\mathrm{Ge}$ atoms, i.e., given the stoichiometry, $n_{\mathrm{GeGe}}$ and $n_{\mathrm{TeGe}}$ dominate. We find $n_{\mathrm{Ge}}=3.99$ and $n_{\mathrm{Te}}=2.48$, or numbers which are found to be either close to the experimental determination [9] or close to some of the RMC determinations of $n_{\mathrm{Ge}}$. While $\mathrm{Ge}$ atoms are predominantly in a fourfold coordination, Te has a slightly higher coordination number than what would be expected from the simple application of the $8-\mathcal{N}$ bonding rule, where $\mathcal{N}$ represent the number of outer-shell $\mathrm{s}$ and $\mathrm{p}$ electrons. It should be remembered that this rule holds for lighter chalcogenides ( $S$, Se [63]). Here, $n_{\mathrm{Te}}$ is substantially reduced when compared to the simulations without the dispersive correction (here $n_{\mathrm{Te}}=2.58$ and 2.68 for PBE and PBEsol, respectively). Previously, it has been found that $n_{\mathrm{Te}}=3.30$ [20] but by using a different cut-off value $r_{m}$. 
TABLE V. Proportion of different coordination units in amorphous GeTe. The fraction of the central atom $(\mathrm{Ge}, \mathrm{Te})$ that has $i$ neighbors is given in bold font. The fraction of homopolar bonds among a given species $(0,1,2, \ldots)$ is given in the next columns. The cut-off distance for the calculation of the coordination numbers has been taken at the minimum $r_{m}=3.2 \AA$ of the pair distribution functions.

\begin{tabular}{lccccccc}
\hline \hline & & 0 & 1 & 2 & 3 & 4 & 5 \\
\hline $\mathrm{Ge}^{\mathrm{III}}$ & $\mathbf{8 . 2 5}$ & 3.94 & 3.43 & 0.04 & & & \\
$\mathrm{Ge}^{\mathrm{IV}}$ & $\mathbf{8 1 . 1 6}$ & 6.73 & 20.25 & 31.14 & 21.60 & 1.42 & - \\
$\mathrm{Ge}^{\mathrm{V}}$ & $\mathbf{1 0 . 1 4}$ & 0.84 & 1.47 & 3.26 & 2.32 & 2.21 & 0.01 \\
$\mathrm{Te}^{\mathrm{I}}$ & $\mathbf{0 . 6 5}$ & 0.63 & 0.02 & - & - & - & - \\
$\mathrm{Te}^{\mathrm{II}}$ & $\mathbf{5 7 . 0 9}$ & 48.58 & 8.48 & 0.032 & - & - & - \\
$\mathrm{Te}^{\mathrm{III}}$ & $\mathbf{3 9 . 6 9}$ & 28.79 & 8.02 & 3.15 & & - & - \\
$\mathrm{Te}^{\mathrm{IV}}$ & $\mathbf{2 . 2 6}$ & 0.87 & 0.39 & 0.95 & 0.05 & & - \\
\hline \hline
\end{tabular}

An inspection of the corresponding pair-correlation functions (Fig. 7) shows that PBE and PBEsol lead to a function $g_{\mathrm{GeTe}}(r)$ that has a somewhat larger amplitude for the first peak and a value $g_{\mathrm{GeTe}}\left(r_{m}\right)$ that is slightly larger as the vdW calculation. As seen from Table IV, the difference in Te coordination between the different DFT models also arises from increased contributions to $n_{\mathrm{GeTe}}$. One should also notice that even the RMC models display quite different coordination numbers, and this is a consequence of the obtained partial correlation functions using this technique, especially $g_{\mathrm{TeTe}}(r)$, which is found to exhibit a prominent homopolar peak in Ref. [49], whereas it is significantly reduced in Ref. [50].

Finally, we calculate (Table V) the fraction of $r$-fold coordinated species in the DFT-D2 amorphous GeTe. Results show that germanium atoms have a non-negligible fraction of coordination defects which are essentially $3-\left(\mathrm{Ge}^{\mathrm{III}}, 8.25 \%\right)$ and fivefold $\left(\mathrm{Ge}^{\mathrm{V}}, 10.14 \%\right)$ coordinated, the dominant motif remaining, of course, $\mathrm{Ge}^{\mathrm{IV}}(81.16 \%)$. Among such species, one should notice that such $\mathrm{Ge}^{\mathrm{IV}}$ have a larger amount of atoms with two homopolar Ge-Ge bonds (31.14\%), whereas only a small fraction of Ge atoms are fully heteropolar $(100 \% \mathrm{Ge}-\mathrm{Te}$, i.e., $6.73 \%$ ). Concerning the Te-based species, because of the increased coordination number (as compared to a perfect 8- $\mathcal{N}$ rule) which has been found to be $\mathrm{n}_{\mathrm{Te}}=2.48$ (see Table IV), one has two dominant motifs, $\mathrm{Te}^{\mathrm{II}}$ and $\mathrm{Te}^{\mathrm{III}}$, whose population is $57.09 \%$ and $39.69 \%$, respectively. These motifs appear to be mostly linked to Ge atoms $(48.58 \%$ over $57.09 \%$ of $\mathrm{Te}^{\mathrm{II}}$ ), as also indirectly revealed by the barely observable homopolar Te-Te prepeak in the partial correlation function $g_{\mathrm{TeTe}}(r)$ (Fig. 7).

From such statistics (Table V), we also calculate the fraction of $\mathrm{Ge}-\mathrm{Ge}, \mathrm{Ge}-\mathrm{Te}$, and $\mathrm{Te}-\mathrm{Te}$ bonds, and results are given in Table VI. It is found that the network is predominantly made of Ge-Ge and Ge-Te bonds, the fraction of Te-Te bonds being rather small, i.e., less than $10 \%$. We, furthermore, find a ratio of Ge-Ge to Ge-Te bonds that is balanced $(\simeq 2: 2)$, almost consistent with findings from RMC [49,50,58]. These fractions are found to be substantially altered when the correction due to dispersion forces is not taken into account, i.e., one finds a fraction of Ge-Ge bonds that is now reduced (38.5\%-39.6\%) for both PBE and PBEsol functionals, as also detected from
TABLE VI. Proportion of bonds in amorphous GeTe (in \%) for the three structure models. Results from RMC models $[49,50]$ are also given for comparison.

\begin{tabular}{lccc}
\hline \hline & Ge-Ge & Ge-Te & Te-Te \\
\hline DFT-D2 (vdW) & 45.1 & 45.3 & 9.6 \\
PBEsol & 39.6 & 52.4 & 8.0 \\
PBE & 38.5 & 58.8 & 2.7 \\
RMC [49] & 39.2 & 50.0 & 9.7 \\
RMC [50] & 23.0 & 73.3 & 3.7 \\
\hline \hline
\end{tabular}

the reduced amplitude of the first (homopolar) peak in $g_{\mathrm{GeGe}}(r)$ (Fig. 7). Given that the increased presence of Ge-Ge bonds has been linked with an growing presence of tetrahedral motifs $[19,48]$, one is led to believe that the change in bonding statistics (from PBE and PBEsol to vdW) should, indeed, impact the fraction of tetrahedra present in the structure, as discussed below.

\section{Increased tetrahedral character \\ 1. Global analysis}

Figure 8 shows the Ge-Te-Ge and Te-Ge-Te bond angle distributions (BAD) for the three structural models. The former indicates that $\mathrm{Te}$ atoms involve an angle of about $90^{\circ}$, consistent with previous findings using similar electronic schemes [16,20,21], the presence of dispersion forces leading only to a global sharpening of the distribution, which is an indication of a better resolved geometry. The latter (Te-Ge-Te) shows that with the presence of the Grimme correction, the peak of the Te-Ge-Te distribution shifts to larger angles, approximately from $\simeq 98^{\circ}$ to $102^{\circ}$, a feature already anticipated from the bond length reduction (see above). In fact, in oxides it is well known that application of pressure leads to the transformation of a $\mathrm{sp}^{3}$ tetrahedral into an octahedral geometry $[11,67]$. This transformation is accompanied by an increase of corresponding bond lengths [10]. In the present GeTe system, the acknowledged reduction of the Ge-Te bond
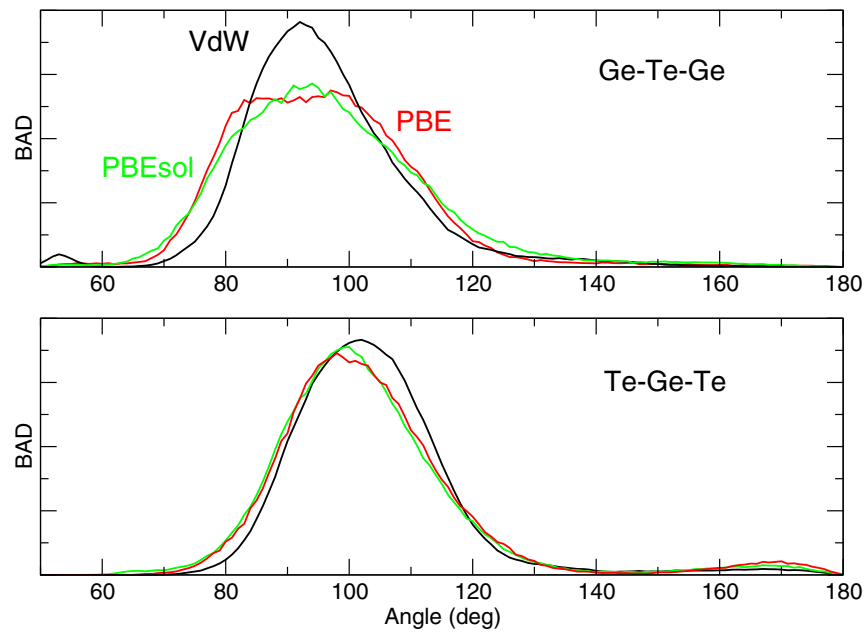

FIG. 8. Computed bond angle distribution Te-Ge-Te and Ge-Te$\mathrm{Ge}$ in amorphous GeTe. 


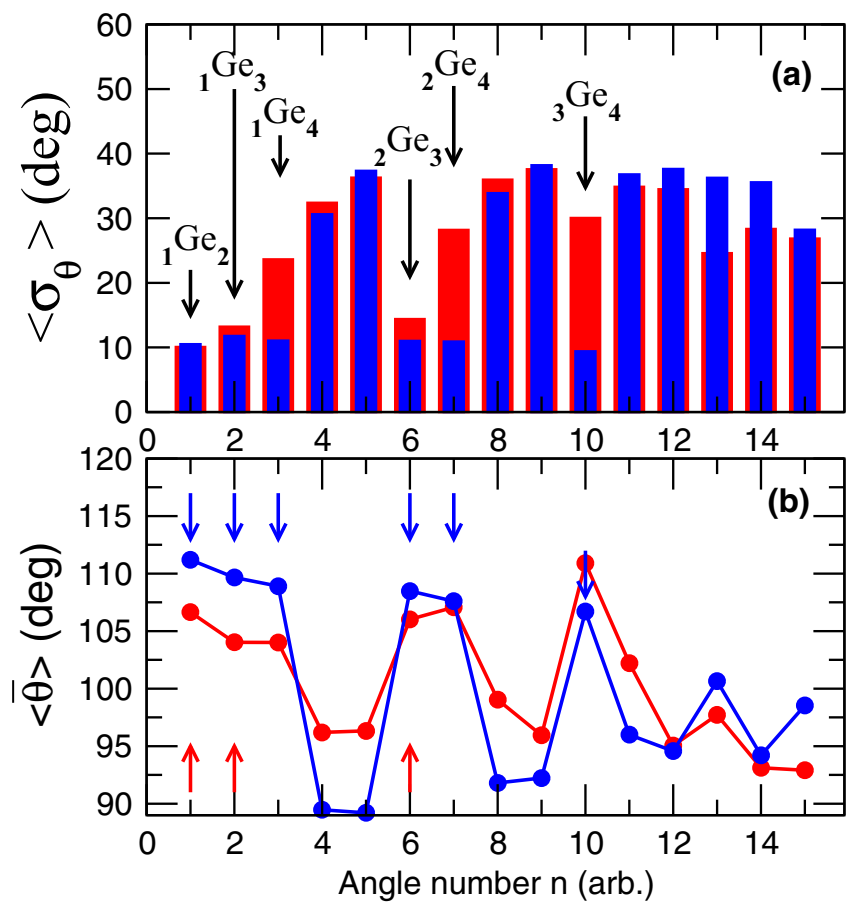

FIG. 9. (a) Standard deviation $\left\langle\sigma_{\theta}\right\rangle$ for arbitrary angle numbers $n$ around Ge atoms in DFT-D2 (vdW) simulated amorphous GeTe split into two categories: Ge atoms having six low $\left\langle\sigma_{\theta}\right\rangle$ (blue), and Ge atoms not having such six $\left\langle\sigma_{\theta}\right\rangle$ (red). Brackets indicate system averages. The arrows indicate the relevant angles ${ }_{k} \mathrm{Ge}_{m}(n)$ serving for the discussion. Here, $m$ and $k<m$ are the Ge neighbors, labeled according to their distance with respect to the central Ge atom. (b) Corresponding Ge-centered angles $\langle\bar{\theta}\rangle$. Colored arrows indicate angles which can be considered as rigid [because of (a)].

length (Table III) leads, therefore, to an increased tetrahedral character for the Ge atoms. This statement can be made more quantitative as discussed next.

\section{Determination of the tetrahedral fraction}

We have mentioned that the reduction of the Ge-Te bond length will impact the Ge geometry. To quantify the fraction $\eta_{T}$ of germanium tetrahedra, we use a recently introduced method $[34,68]$ to determine without any ambiguity the fraction of $\mathrm{Ge}$ tetrahedral in the structure.

Note that the method is based on the enumeration of angular topological constraints [69]. Rather than focusing on the typical angles between two well-defined geometries [20] (tetrahedral $109^{\circ}$ and defect octahedral $90-100^{\circ}$ ) or on a bond length argument [21] that gives rise to some uncertainties, we focus on angular excursions that are computed on-the-fly. One follows a set of $N(N-1) / 2$ angles defined by the $N$ first neighbors around a central Ge. Over the simulated trajectory (i.e., with time), these individual angles define a partial bond angle distribution out of which a mean $\bar{\theta}$ and a standard deviation $\sigma_{\theta}$ can be computed for each Ge of the network. If the number of low standard deviations $\sigma_{\theta}$ around a $\mathrm{Ge}$ atom is six, a tetrahedron is identified because this geometry is defined by six rigid angles [69] that give rise to corresponding low angular standard deviations. On system average, such typical tetrahedral angles are well defined [Fig. 9(a)] and lead to a

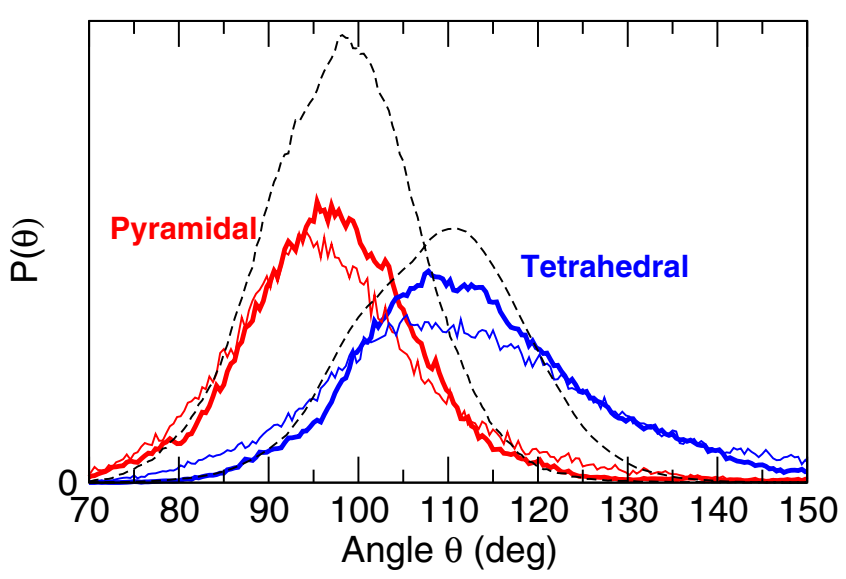

FIG. 10. Resulting bond angle distribution of identified tetrahedral (blue) and pyramidal (red) geometries in DFT-D2 (vdW, thick lines) and PBEsol (thin lines) simulated amorphous GeTe. The PBE simulation (not shown) is close to PBEsol. The broken black lines correspond to reference compounds having a perfect pyramidal $\left(\mathrm{As}_{2} \mathrm{Se}_{3}\right)$ [70] or tetrahedral geometry $\left(\mathrm{Ge}_{20} \mathrm{Se}_{80}\right)$ [63].

corresponding angle that is, in fact, very close to the tetrahedral angle of $109^{\circ}$ [Fig. 9(b)].

When such an analysis is performed on the three simulations of amorphous GeTe (Fig. 9), one obtains a fraction of Ge tetrahedra $\eta_{T}=64.7 \%, \eta_{T}=43.8 \%$, and $\eta_{T}=41.2 \%$ for the vdW, PBEsol and PBE simulations, respectively. Notice that for the regular PBE simulations a calculation of $\eta_{T}$ using a criteria on long Ge-Te bonds [21] found among the bimodal fourth Ge neighbor distribution leads to a value (19.6\%) that is compatible with the one determined either for other alloys $\left(\mathrm{Ge}_{1} \mathrm{Sb}_{2} \mathrm{Te}_{4}\right.$ [21]) or for the GeTe system using different criteria for the enumeration of tetrahedra (see [34] for a review of methods). Once the Ge tetrahedra is identified, it is then possible to split the corresponding Ge-centered bond angle distribution into a contribution arising from the tetrahedra and a contribution due to a residual (or nontetrahedral) geometry, found to be close to that of a pyramid (red curves in Fig. 10). In fact, the corresponding BAD is found to be centered at an angle of $98^{\circ}$, similarly to the Se-As-Se BAD in a- $\mathrm{As}_{2} \mathrm{Se}_{3}$ for which the pyramidal geometry of arsenic can be unambiguously identified [70]. The Grimme dispersion thus leads to models of amorphous GeTe that contain a much larger fraction of tetrahedral germanium. It should also be noted that the substantial decrease of $\eta_{T}$ when dispersion forces are absent is also due to a strong reduction of the fraction of fourfold $\mathrm{Ge}\left(\mathrm{Ge}^{\mathrm{IV}}\right.$, see Table V), which decreases from $81.16 \%$ to $60.72 \%$ when one changes from DFT-D2 to PBEsol (and $67.9 \%$ for PBE).

The question of the nature of fourfold germanium geometry is also related to the way the material is produced. Experimental data are usually extracted from as-deposited samples (see Table I) using sputtering setups. Therefore the way such samples are produced differ substantially from amorphous melt-quenched numerical samples. Akola and coworkers [52] have recently shown that a structural DFT model generated by deposition does indeed show a larger fraction of tetrahedral $\mathrm{Ge}$ atoms, induced by a larger number of $\mathrm{Ge}-\mathrm{Ge}$ 
bonds. This indicates that differences in structural properties emerge from the preparation conditions, and these also impact the crystallization kinetics [2]. Since the simulations of asdeposition were performed with PBEsol, it would certainly be of interest to probe the combined effect of dispersion forces and the numerical preparation technique.

\section{Reduced ABAB ring statistics}

It has been stressed that the important crystallization speed achieved during the amorphous-to-crystal transformation results from the presence of so-called $A B A B$ rings $(A=G e$, $B=T e)$. These represent four-membered loops which contain perfect bond alternation, i.e., only heteropolar Ge-Te bonds are able to serve as the basic building block for the growth of the rocksaltlike crystalline phase of GeTe. Akola and Jones $[20,71,72]$ and other authors [17,29] have shown, in fact, that the onset of crystalline order is strongly correlated with the growth of such ABAB motifs. Most of the ring analysis, however, has been performed on structures containing the usual flaws of the regular DFT scheme, i.e., bond length mismatch resulting in only a fair agreement of the simulated structure functions in real or reciprocal space with the experimental counterparts.

The present ring calculation uses an algorithm that is mostly based on the King [73] and Franzblau [74] shortest path search. The statistics is computed [75,76] up to rings of size $n=10$ atoms, and a cutoff distance of $3.2 \AA$ has been used for all atomic pairs. Minor changes are found under a cut-off change of $\pm 0.1 \AA$. Figure 11 represents the number of rings found in the structure for the three simulation schemes, PBE (a), PBEsol (b), and vdW (c). First, one should note that, in contrast with oxide network formers (e.g., $\mathrm{SiO}_{2}$ ), having $100 \%$ heteropolar bonds and only even sized rings [77,78], tellurides and can have both even- and odd-sized rings because of the presence of the homopolar bonds, this situation being also encountered in selenides and sulfides $[63,70,79]$.

We find that the ring distribution of amorphous GeTe is dominated by five- and sixfold rings (Fig. 12). This situation is true for all simulations, the PBEsol showing an increased fraction of large rings, typically 8 - and 10-membered loops, whereas the PBE has the largest fivefold ring fraction, the obtained distribution being also similar to the one of the RMC model of Stellhorn et al. [50]. We also note that the PBE simulation (panel a) has the largest tendency to form ABAB rings, as also found in similar simulations [71,72]. However, $\mathrm{ABAB}$ motifs seem to be altered by the presence of dispersion forces. In fact, in a regular DFT scheme (no vdW), ABAB are found to substantially contribute to the fraction of fourmembered rings but they are found to be reduced in the vdW models. Furthermore, larger ABAB rings are also impacted by the presence of dispersion forces because they are absent in eight- and ten-membered loops but present in small amounts in the PBEsol simulation. The same conclusion has been drawn for Ge-Sb-Te liquids [46], and a study as a function of the cut-off value showed that the conclusion is not altered with a change in $r_{m}$.

Overall, the present ring distribution is consistent with that found from the most recent RMC model exhibiting a similar
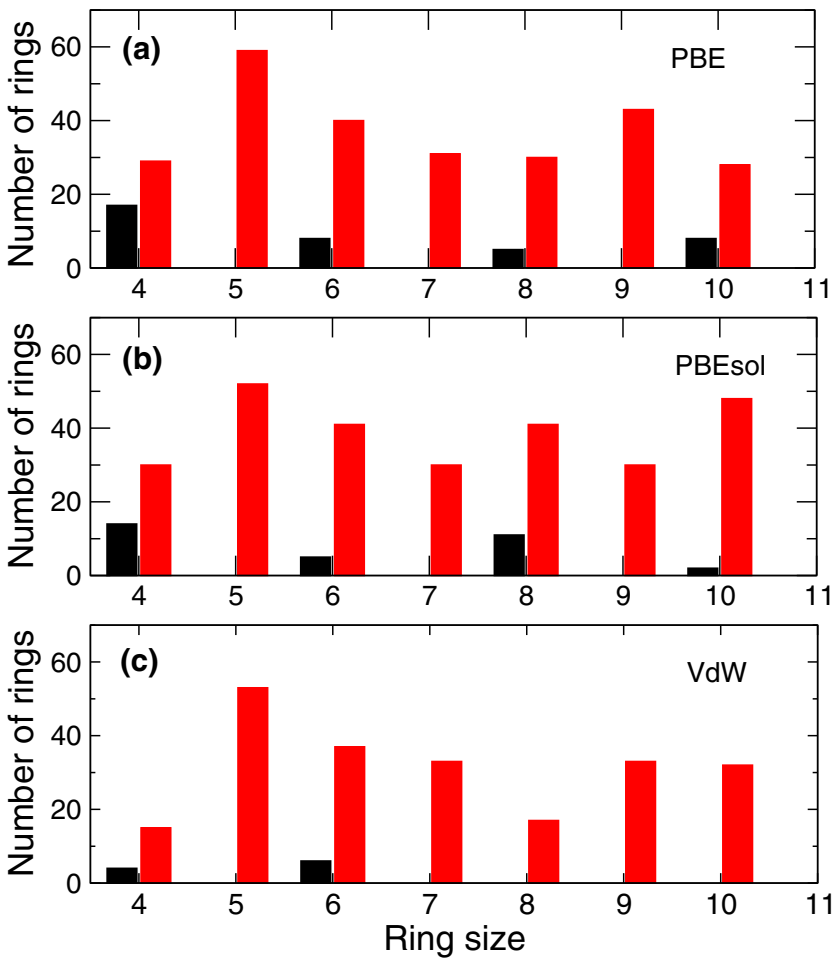

FIG. 11. Calculated ring statistics (red) in amorphous GeTe and corresponding ABAB statistics (black) for PBE (a), PBEsol (b), and DFT-D2 (vdW) (c) simulations.

distribution [50]. The ratios for the $\mathrm{ABAB}$ rings are also found to be very close, i.e., we find that $\mathrm{ABAB}$ motifs represents about $27 \%$ of the four-membered ring distribution (i.e., four $\mathrm{ABAB}$ rings over a total population of 15 four-membered rings). The fraction of the most present rings is 0.185 ring/atom and 0.265 ring per atom for five- and sixfold rings, respectively.

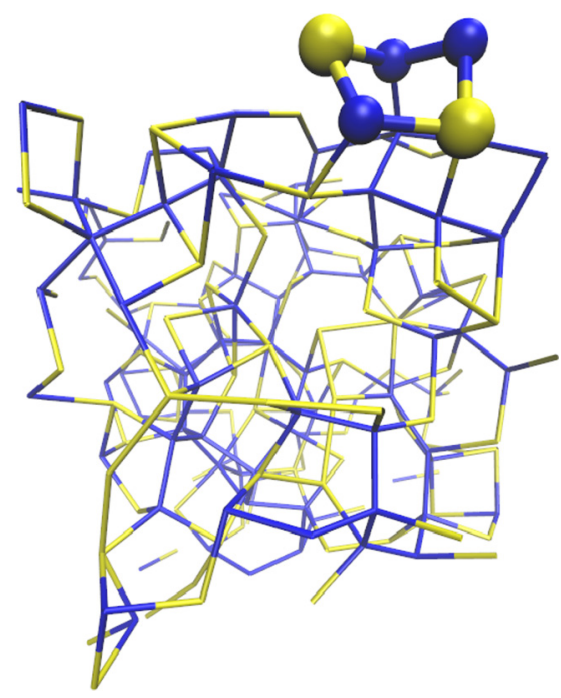

FIG. 12. Snapshot of an amorphous GeTe configuration with distorted five-membered rings represented. 


\section{CONCLUSION}

Amorphous materials belonging to the $\mathrm{Ge}_{x} \mathrm{Te}_{1-x}$ binary system and particularly the GeTe one represent archetypal networks that can be used for an increasing number of applications in optoelectronics. The understanding of the structural, vibrational, thermal, and electronic properties of this material and of related ones is an important issue given that many such applications benefit from the low band gaps, allowing for dedicated electronic functionalities.

Here we have studied the structure of this amorphous GeTe by using CPMD simulations in conjunction with dispersive interactions (DFT-D2) based on the Grimme corrections that handles the effect of polarizability in a phenomenological fashion. A systematic structural analysis has been performed, and results have been compared with two benchmark systems having undergone the same thermal history but without the dispersive correction. These correspond to the PBE functional and to an improved scheme (PBEsol). With respect to these systems and to previous studies on the same material, the present study marks a substantial improvement when the result from simulations is compared to experiments on structural properties accessed from XRD. This is especially appealing from the agreement obtained in the reproduction of the pair-correlation function in real space and the structure factor in reciprocal space. Results are compared to a typical benchmark system using DFT without any corrections (PBE and PBEsol). As a general result, DFT-D2 leads to typical bond distances that contribute to the first peak of the pair correlation function $\mathrm{g}(\mathrm{r})$, identified with contributions coming from both $\mathrm{Ge}-\mathrm{Te}$ and Te-Te bonds. This improved scheme also shows that the coordination num- bers are decreased with respect to previous findings, a result that mostly arises from the fact that the first peak in $\mathrm{g}(\mathrm{r})$ is reproduced with an unprecedented accuracy. We have also compared the numerical results to more recent experimental findings from EXAFS and AXS which represent additional constraints for modeling schemes. Another important result is that the bond distance reduction leads to an increased population of tetrahedral Ge in the amorphous DFT-D2 system, which represents about $64.7 \%$, while also suggesting that DFT simulations without dispersion forces systematically lead to fractions of tetrahedra that are much smaller. The present findings are, furthermore, consistent with a recent investigation on Te-rich Ge-Te glasses [34] which showed that the same incorporation of Grimme corrections led to a population of Ge tetrahedra in agreement with experimental measurements from Mössbauer spectroscopy. This increased tetrahedral fraction from more accurate structural models might provide some renewed credit to EXAFS-based models [9] proposing a large number of tetrahedra in the amorphous phase. It would be very interesting to check how the dynamics and crystallization kinetics are modified once these more realistic structural models are assessed.

\section{ACKNOWLEDGMENTS}

Support from Agence Nationale de la Recherche (ANR) (Grant No. ANR-11-BS08-0012) is gratefully acknowledged. GENCI (Grand Equipement National de Calcul Intensif) and Université de Champagne-Ardenne (Romeo) is acknowledged for supercomputing access.
[1] S. Ovshinsky, Phys. Rev. Lett. 21, 1450 (1968).

[2] Phase Change Materials and Applications, edited by S. Raoux and M. Wuttig (Springer, Berlin, 2008).

[3] S. Raoux, W. Wełnic, and D. Ielmini, Chem. Rev. 110, 240 (2010).

[4] C. Vigreux, E. Barthélémy, L. Bastard, J.-E. Broquin, S. Ménard, M. Barillot, G. Parent, and A. Pradel, Opt. Mater. Express 1, 357 (2011).

[5] P. Lucas, Z. Yang, M. K. Fah, T. Luo, S. Jiang, C. BoussardPledel, M.-L. Anne, and B. Bureau, Opt. Mater. Express 3, 1049 (2013).

[6] W. Welnic, A. Pamungkas, R. Detemple, C. Steimer, S. Blügel, and M. Wuttig, Nat. Mater. 5, 56 (2006).

[7] H. F. Hamman, M. O’Boyle, Y. C. Martin, M. Rooks, and H. K. Wickramasinghe, Nat. Mater. 5, 383 (2006).

[8] X. Zhou, Y. Du, J. K. Behera, Z. Song, and R. E. Simpson, ACS Appl. Mater. Interfaces 8, 20185 (2016).

[9] A. V. Kolobov, P. Fons, A. I. Frenkel, A. L. Ankudinov, J. Tominaga, and T. Uruga, Nat. Mater. 3, 703 (2004).

[10] J. P. Itie, A. Polian, G. Calas, J. Petiau, A. Fontaine, and H. Tolentino, Phys. Rev. Lett. 63, 398 (1989).

[11] M. Micoulaut, J. Phys.: Condens. Matter 16, L131 (2004).

[12] D. A. Baker, M. A. Paesler, G. Lucovsky, S. C. Agarwal, and P. C. Taylor, Phys. Rev. Lett. 96, 255501 (2006).
[13] J. Y. Raty, V. V. Godlevsky, J. P. Gaspard, C. Bichara, M. Bionducci, R. Bellissent, R. Céolin, J. R. Chelikowsky, and P. Ghosez, Phys. Rev. B 65, 115205 (2002).

[14] J. Akola and R. O. Jones, Phys. Rev. Lett. 100, 205502 (2008).

[15] S. Gabardi, S. Caravati, G. C. Sosso, J. Behler, and M. Bernasconi, Phys. Rev. B 92, 054201 (2015).

[16] S. Caravati, M. Bernasconi, and M. Parrinello, J. Phys.: Condens. Matter 22, 315801 (2010)

[17] J. Hegedüs and S. R. Elliott, Nat. Mater. 7, 399 (2008).

[18] W. Zhang, V. L. Deringer, R. Dronskowski, and R. Mazzarello, MRS Bull. 40, 856 (2015).

[19] J.-Y. Raty, W. Zhang, J. Luckas, C. Chen, R. Mazzarello, C. Bichara, and M. Wuttig, Nat. Commun. 6, 7467 (2015).

[20] J. Akola and R. O. Jones, Phys. Rev. B 76, 235201 (2007).

[21] J.-Y. Raty, C. Otjacques, J. P. Gaspard, and C. Bichara, Solid State Sci. 12, 193 (2010).

[22] J. Kalikka, J. Akola, R. O. Jones, S. Kohara, and T. Usuki, J. Phys.: Condens. Matter 24, 015802 (2012).

[23] S. Caravati, M. Bernasconi, T. D. Kuehne, M. Krack, and M. Parrinello, Appl. Phys. Lett. 91, 171906 (2007).

[24] R. Mazzarello, S. Caravati, S. Angioletti-Uberti, M. Bernasconi, and M. Parrinello, Phys. Rev. Lett. 104, 085503 (2010).

[25] M. Krbal, A. V. Kolobov, P. Fons, K. V. Mitrofanov, Y. Tameroni, J. Hegedüs, S. R. Elliott, and J. Tominaga, Appl. Phys. Lett. 102, 111904 (2013). 
[26] K. Shportko, S. Kremers, M. Woda, D. Lencer, J. Robertson, and M. Wuttig, Nat. Mater. 7, 653 (2008).

[27] J. A. Dixon and S. R. Elliott, Appl. Phys. Lett. 104, 141905 (2014).

[28] P. Jóvári, I. Kaban, J. Steiner, B. Beuneu, A. Schöps, and M. A. Webb, Phys. Rev. B 77, 035202 (2008).

[29] S. Kohara et al., Appl. Phys. Lett. 89, 201910 (2006).

[30] C. Steimer, V. Coulet, W. Wełnic, H. Dieker, R. Detemple, C. Bichara, B. Beuneu, J. P. Gaspard, and M. Wuttig, Adv. Mater. 20, 4535 (2008).

[31] C. Massobrio, M. Celino, P. S. Salmon, R. A. Martin, M. Micoulaut, and A. Pasquarello, Phys. Rev. B 79, 174201 (2009).

[32] M. Micoulaut, A. Kachmar, and Th. Charpentier, Phys. Status Solidi B 249, 1890 (2012).

[33] E. Cho, J. Im, C. Park, W. J. Son, D. H. Kim, H. Horii, J. Ihm, and S. Han, J. Phys.: Condens. Matter 22, 205504 (2010).

[34] M. Micoulaut, K. Gunasekera, S. Ravindren, and P. Boolchand, Phys. Rev. B 90, 094207 (2014).

[35] S. Caravati and M. Bernasconi, Phys. Status Solidi B 252, 260 (2015).

[36] M. Micoulaut, R. Vuilleumier, and C. Massobrio, Phys. Rev. B 79, 214205 (2009).

[37] S. LeRoux, M. Boero, M. Micoulaut, A. Zeidler, P. S. Salmon, and C. Massobrio, Phys. Rev. B 84, 134203 (2011).

[38] C. Massobrio, M. Micoulaut, and P. S. Salmon, Solid State Sci. 12, 199 (2010).

[39] K. Y. Kim, D. Y. Cho, B. Cheong, D. Kim, H. Horii, and S. Han, J. Appl. Phys. 113, 134302 (2013).

[40] A. Bouzid, C. Massobrio, M. Boero, G. Ori, K. Sykina, and E. Furet, Phys. Rev. B 92, 134208 (2015).

[41] S. Grimme, J. Comput. Chem. 27, 1787 (2006).

[42] Z. Ren, A. A. Taskin, S. Sasaki, K. Segawa, and Y. Ando, Phys. Rev. B 85, 155301 (2012).

[43] B. Sa, J. Zhou, R. Ahuja, and Z. Sun, Comput. Mater. Sci. 82, 66 (2014).

[44] M. Micoulaut, J. Chem. Phys. 138, 061103 (2013).

[45] M. Micoulaut, M.-V. Coulet, A. Piarristeguy, M. R. Johnson, G. J. Cuello, C. Bichara, J.-Y. Raty, H. Flores-Ruiz, and A. Pradel, Phys. Rev. B 89, 174205 (2014).

[46] H. Flores-Ruiz, M. Micoulaut, M.-V. Coulet, A. A. Piarristeguy, M. R. Johnson, G. J. Cuello, and A. Pradel, Phys. Rev. B 92, 134205 (2015).

[47] J. Akola, R. O. Jones, S. Kohara, T. Usuki, and E. Bychkov, Phys. Rev. B 81, 094202 (2010).

[48] G. E. Ghezzi, J.-Y. Raty, S. Maitrejean, A. Roule, E. Elkaim, and F. Hippert, Appl. Phys. Lett. 99, 151906 (2011).

[49] A. Piarristeguy, M. Micoulaut, R. Escalier, P. Jovari, I. Kaban, J. van Eijk, J. Luckas, S. Ravindren, P. Boolchand, and A. Pradel, J. Chem. Phys. 143, 074502 (2015).

[50] J. R. Stellhorn, S. Hosokawa, W.-C. Pilgrim, N. Blanc, N. Boudet, H. Tajiri, and S. Kohara, Phys. Status Solidi 253, 1038 (2016).

[51] N. Yamada and T. Matsunaga, J. Appl. Phys. 88, 7020 (2000).
[52] J. Akola, J. Larrucea, and R. O. Jones, Phys. Rev. B 83, 094113 (2011).

[53] S. Caravati, M. Bernasconi, T. D. Kühne, M. Krack, and M. Parrinello, J. Phys.: Condens. Matter 21, 255501 (2009).

[54] J. J. Rehr and R. C. Albers, Phys. Rev. B 41, 8139 (1990).

[55] G. Ferlat, J. C. Soetens, A. S. Miguel, and P. A. Bopp, J. Phys.: Condens. Matter 17, S145 (2005).

[56] J.-Y. Raty, C. Otjacques, R. Peköz, V. Lordi, and C. Bichara, in Molecular Dynamics Simulations of Disordered Materials, edited by C. Massobrio, J. Du, M. Bernasconi, and P. Salmon (Springer, Heidelberg, 2015).

[57] P. Noé, C. Sabbione, N. Castellani, G. Veux, G. Navarro, V. Sousa, F. Hippert, and F. d'Acapito, J. Phys. D: Appl. Phys. 49, 035305 (2016).

[58] J. M. Van Eijk, Structural analysis of phase change materials using x-ray absorption measurements, Ph.D. thesis, RWTH Aachen University, 2010.

[59] S. Hosokawa, Y. Kawakita, W.-C. Pilgrim, and F. Hensel, J. Non-Cryst. Solids 293-295, 153 (2001).

[60] S. Hosokawa, I. Oh, M. Sakurai, W.-C. Pilgrim, N. Boudet, and J.-F. Bérar, Phys. Status Solidi 206, 1736 (2009).

[61] S. Hosokawa, I. Oh, M. Sakurai, W.-C. Pilgrim, N. Boudet, J.-F. Bérar, and S. Kohara, Phys. Rev. B 84, 014201 (2011).

[62] Y. Waseda, Novel Application of Anomalous X-ray Scattering for Structural Characterization of Disordered Materials (SpringerVerlag, Heidelberg, 1984).

[63] M. Micoulaut, A. Kachmar, M. Bauchy, S. Le Roux, C. Massobrio, and M. Boero, Phys. Rev. B 88, 054203 (2013).

[64] Y. Maeda and M. Wakagi, Jap. J. Appl. phys. 30, 101 (1991).

[65] K. Hirota, K. Nagino, and G. Ohbayashi, J. Appl. Phys. 82, 65 (1997).

[66] P. Jávári, A. Piarristeguy, R. Escalier, I. Kaban, J. Bednarcik, and A. Pradel, J. Phys.: Condens. Matter 25, 195401 (2013).

[67] M. Micoulaut, Y. Guissani, and B. Guillot, Phys. Rev. E 73, 031504 (2006).

[68] K. Gunasekera, P. Boolchand, and M. Micoulaut, J. Appl. Phys. 115, 164905 (2014).

[69] M. Micoulaut, Adv. Phys. X 1, 147 (2016).

[70] M. Bauchy, A. Kachmar, and M. Micoulaut, J. Chem. Phys. 141, 194506 (2014).

[71] J. Kalikka, J. Akola, J. Larrucea, and R. O. Jones, Phys. Rev. B 86, 144113 (2012).

[72] J. Akola and R. O. Jones, Phys. Rev. B 79, 134118 (2009).

[73] V. King, Nature (London) 213, 1112 (1967).

[74] D. S. Franzblau, Phys. Rev. B 44, 4925 (1991).

[75] S. Le Roux and P. Jund, Comput. Mater. Sci. 49, 70 (2010).

[76] S. Le Roux and P. Jund, Comput. Mater. Sci. 50, 1217 (2011).

[77] X. Yuan and A. N. Cormack, Comp. Mater. Sci. 24, 343 (2002).

[78] M. Micoulaut, X. Yuan, and L. W. Hobbs, J. Non-Cryst. Solids 353, 1951 (2007).

[79] S. Chakraborty and P. Boolchand, J. Phys. Chem. B 118, 2249 (2014). 